\title{
Ovarian and Uterine Blood Flow Indices in Patients with Unexplained Infertility Undergoing ICSI and their Relation to Clinical Pregnancy Rate
}

\section{Original Article}

\author{
Ahmed Mohamed Ibrahim, Mohamed El-Mandooh Mohamed, Haitham Fathy \\ Mohammed Gad, Heba Abd Alfatah Wafa Alhariri
}

Department of Obstetrics and Gynecology, Faculty of Medicine, Ain-Shams University, Cairo, Egypt

\begin{abstract}
Background: Infertility is defined by the World Health Organisation (WHO) as a disease of the reproductive system defined by the failure to achieve a clinical pregnancy after 12 months or more of regular unprotected sexual intercourse and is estimated to affect as many as 48.5 million couples worldwide. Unexplained infertility (UI) constitutes an important portion of the infertility reasons (up to $30 \%$ ) and is diagnosed when the causes of male or female infertility remains unknown.

Aim: The aim of the study was to compare ovarian, uterine artery and subendometrial vessels resistance by measuring PI and RI of patients diagnosed as UI undergoing ICSI in the day of embryo transfer and control group diagnosed as fertile by having at least one livebirth in peri-implantation period from day 19 to day 21.

Materials and Methods: The current study is a prospective case control study, which was conducted at the Assisted Reproduction Technology unit of Ain Shams University Maternity Hospital during the period from September 2017 to July 2019. The current study included two groups of patients one group is unexplained infertility attending the Assisted Reproduction Unit and the other is control fertile group each group consists of 51 women.

Results: There was statistically significant difference between the unexplained infertility group and control fertile group as regard Uterine and subendometrial artery PI, RI and ovarian artery RI in which resistance indexes was significantly higher in the UI group than control group. The cut off values of PI, RI between unexplained infertility and fertile group are $\geq 1.88, \geq 0.8$ for Uterine artery, and $\geq 0.87, \geq 0.53$ for subendometrial arteries, and $\geq 0.59$ for ovarian artery RI. Among the unexplained infertility undergoing ICSI patients the pregnancy rate is $23.5 \%$. There was no statistically significant difference between the two groups (pregnant group and non-pregnant group) as regard the age, BMI, duration of infertility or the basal hormonal profile.

Conclusion: Doppler parameters are useful tools to assess endometrial receptivity in unexplained infertility patients undergoing ICSI. This study found that Uterine, Ovarian, subendometrial arteries plasticity index (PI) and resistance index RI measured on the day of embryo transfer higher than fertile women and have value in judging endometrial receptivity and predict the final outcome of IVF/ ICSI- ET. Uterine, ovarian and subendometrial blood flow assessed by measuring PI, RI of these arteries was impaired in unexplained infertility patients compared to fertile women. Also, uterine arteries PI, ovarian and subendometrial arteries PI, RI differ in pregnancy outcome in unexplained infertility patients undergoing ICSI.
\end{abstract}

Key Words: ICSI, Uterine blood flow indices, unexplained infertility

Received: 06 December 2019, Accepted: 09 February 2020

Corresponding Author: Heba Abd Alfatah Wafa Alhariri, Department of Obstetrics and Gynecology, Faculty of Medicine, Ain-Shams University, Egypt, Tel.: 01112340298, E-mail: elhaririheba@gmail.com

ISSN: 2090-7265, August 2020, Vol.10, No. 3

\section{INTRODUCTION}

Infertility is defined by the World Health Organization (WHO) as a disease of the reproductive system defined by the failure to achieve a clinical pregnancy after 12 months or more of regular unprotected sexual intercourse ${ }^{[15]}$ and is estimated to affect as many as 48.5 million couples worldwide $^{[8]}$.

In general, infertility can be attributed to the female partner one third of the time, the male partner one third of the time and both partners in the remaining one third. Estimates of the incidence of various causes of infertility are male $25 \%$, ovulatory such as PCO $27 \%$, tubal/uterine $22 \%$, other $9 \%$ and unexplained $17 \%{ }^{[2]}$.

Unexplained infertility (UI) constitutes an important portion of the infertility reasons (up to $30 \%)^{[10]}$ and is diagnosed when the causes of male or female infertility remains unknown. 
Current infertility treatments such as surgical measures to treat genital tract obstruction or endometriosis, or hormone treatments to restore ovulatory function in women are aimed to reverse the primary causes of infertility. However, in cases where initial treatments are not successful or infertility is unexplained assisted reproductive technology (ART) which includes in vitro fertilization (IVF) and intracytoplasmic sperm injection (ICSI) is the treatment of choice ${ }^{[3]}$.

The success of ICSI-ET predominantly depends on embryo quality, endometrial receptivity and embryo endometrial interface. The endometrial characteristics including endometrial pattern, endometrial blood flow and endometrial thickness (EMT) have been regarded as prognostic factors of IVF-ICSI treatment ${ }^{[13]}$.

The interaction between the embryo and the endometrium is the most important key factor for the success in the treatment of infertile couples. A healthy embryo and a receptive endometrium are needed for a successful implantation. Another important component of the implantation is adequate and effective vascular circulation $^{[9]}$

Doppler parameters have been evaluated as markers of receptivity ${ }^{[6]}$. Doppler ultrasound seems the most popular technique among clinicians because of its reproducibility. Uterine blood flow can be assessed by Doppler ultrasound in the uterine arteries and there is evidence of an association between abnormal uterine artery blood velocities and infertility ${ }^{[12,6]}$. Increase in the resistance of uterine, endometrial and subendometrial arteries is detected in patients with UI during peri-implantation period ${ }^{[5]}$.

Ovarian artery is primarily responsible for the blood supply of the ovaries and the fallopian tubes and indirectly of the endometrium. Resistance changes of ovarian artery have not been studied yet in UI in the presence of a control group $^{[14]}$.

\section{AIM OF THE WORK}

The purpose of this study was to compare ovarian, uterine artery and subendometrial vessels Doppler indices of patients diagnosed as unexplained infertility undergoing ICSI in the peri-implantation period versus control group diagnosed as fertile having at least one live birth.

\section{PATIENTS AND METHODS}

Study design: This was a prospective case control study in which cases were divided into 2 groups; group A included unexplained infertility and group B included control (fertile). It was conducted in the Department of Obstetrics and Gynecology Fertility and IVF Unit and Ultrasound Unit in Ain-Shams University Hospital, Egypt during the period from September 2017 to July 2019.

\section{Inclusion criteria:}

Patients included in this study were divided into two groups.

1- Primary infertile with unexplained infertility (51 patients): Minimum 3 years duration of infertility +/- two trials of intrauterine insemination were done and failed with these criteria:

- Normal ovulation.

- Regular menstrual cycles

- Normal hormonal profile stradiol, midluteal serum estradiol and progesterone, prolactin, thyroid-stimulating hormone, $\mathrm{AMH}$ )

- Patent tubes as checked by:

- Hysterosalpingography (HSG)

- $\quad$ Previous laparoscopy (if done)

- Normal uterus

- $\quad$ Normal ovarian reserve (AFC, hormonal profile)

- Normal semen analysis

- Kruger WHO criteria

2- Control group (51 cases): Women who were presented for a routine gynecological examination (at least one live birth) or in family planning unit for contraception.

Normal gynecological findings.

Normal regular menstrual cycle.

Both groups fulfilled the inclusion criteria:

- $\quad$ The body mass index (BMI) $<30 \mathrm{~kg} / \mathrm{m} 2$.

- $\quad$ Age: 20-37 years.

Patients with the following criteria were excluded from the current study; smoker, lactation, previous ovarian surgery, previous D and C, age $>37$ or $<20$, BMI $>30 \mathrm{~kg} / \mathrm{m} 2$, history of systemic diseases as hypertension and diabetes mellitus, history to receive medical treatments which may influence the pelvic blood flow, male factor infertility and Asherman syndrome which may influence uterine blood flow.

Sample size justification: Sample size was calculated using Stata program, setting the type- 1 error $(\alpha)$ at 0.05 and the power $(1-\beta)$ at 0.9 . The results from a previous study (Zebitay et al., 2015) showed the mean PI for ovarian artery among UI was $1.06 \pm 0.18$ while for control it was $0.96 \pm 0.18$. Calculation according to these values resulted in a minimal sample size of 51 cases per each group (total 102 cases).

\section{Ethical considerations : Study procedure :}

Patients in the unexplained infertility group were subjected to:

\section{1) Verbal consent :}

Consent was obtained from the patients included in the study.

\section{2) History taking :}

Personal history: Name, age, address, occupation, marital history and special habits of medical importance e.g.: smoking. 
Menstrual history; regularity, duration and LMP $\left(1^{\text {st }}\right.$ day of the last menstrual period).

Obstetrics history: Parity, relevant event in obstetric history.

Complain: in the patient's own word.

History of present illness: analysis of complain, duration of infertility, previous investigations.

Medical history, e.g. DM, rheumatologic disease, hypertension and vascular disease.

Surgical history, e.g. Appendectomy, myomectomy, ovarian cystectomy.

Family history.

\section{3) Clinical examination :}

\section{A- General examination :}

Vital data (Blood pressure, pulse and temperature).

The presence of pallor, jaundice or cyanosis.

Calculation of weight, Height and BMI (Body mass index $=$ weight in $\mathrm{Kg}$ /height in $\mathrm{m} 2$ ).

Examination of the extremities.

Chest and Heart examination.

\section{B-Pelvic examination:}

Abdominal and vaginal examinations to access pelvis and exclude any palpable pelvic masses.

\section{2) Laboratory investigation:}

Hormone profile (follicle- stimulating hormone, luteinizing hormone, and estradiol, midluteal serum estradiol and progesterone prolactin, thyroid-stimulating hormone) and semen analysis.

3) Imaging: HSG to assess tubal patency.

\section{4) Ultrasound Technique:}

Ultrasound examination was performed by Dr. Ola Adel Alsergany using 4-9 $\mathrm{MHz}$ endovaginal transducer of the device in the midluteal phase (peri-implantation) at day of ET (Embryo-transfer). The setting condition of the ultrasound for this study was standardized using a frequency at 4-9 $\mathrm{MHz}$, pulse repetition frequency at 1.3 $\mathrm{kHz}$ for uterine and ovarian arteries, $1.1 \mathrm{kHz}$ for spiral artery to evaluate a minimum flow velocity at $5 \mathrm{~cm} / \mathrm{sec}$., gain at 3.4 and wall motion filter at low 1 , harmonic at low and power at $100 \%$. The same setting was used on the machine for all patients. Transverse and sagittal imaging of the uterus and ovaries were performed after insertion of the transducer into the vagina.

Color Doppler examination of the subendometrial arteries was performed within $1-\mathrm{mm}$ area parallel to the myometrium endometrium border. Descending branches of uterine artery were determined at the level of the internal os of cervix uteri. Right and left uterine artery pulsatility index (PI) and resistance index (RI) were calculated and average values were recorded.
Both ovarian arteries were evaluated at ovarian hilus for ovarian arterial study. PI/RI measurements were performed for ovarian artery at ovarian hilus distant from ovarian surface and follicular wall surface. Average values were recorded.

This US examination was done at day of embryo transfer of ICSI technique which is done according to Ain-Shams IVF unit.

\section{ICSI technique:}

The long protocol used, in which the patients started the cycle of treatment by oral contraceptive pills on day 4 of the preceding cycle, down-regulation starts on day 15 of the pills by daily injections of GnRH agonist (Triptorelin Acetate-decapeptyl ${ }^{\circledR} 0.1 \mathrm{mg}$ S.C-FERRING -Germany). Ovarian induction started after the patient being downregulated (as evidenced by U/S picture, no follicle more than $10 \mathrm{~mm}$ in diameter and hormonal profile; serum estradiol level less than $50 \mathrm{pg} / \mathrm{ml}$ ) by hMG (doses are administered and adjusted according to the patients response). Monitoring was performed with serial vaginal ultrasound and plasma estradiol measurement.

Ovulation has been induced by hCG 10,000 IU (2 ampoules of choriomon ${ }^{\circledR} 5000$ IU I.M-IBSA-Switzerland) when three or more follicles in each ovary measured at least $18 \mathrm{~mm}$ in diameter ${ }^{[4]}$.

Transvaginal oocyte retrieval was done then performed under general anaesthesia 34-36 h later. Embryo transfer carried out according to number of embryos and embryo quality on day 3 or day 5 post ovum pick up according to local protocol of IVF unit.

All patients received daily intramuscular progesterone $(100 \mathrm{mg})$ and rectal suppository progesterone $(400 \mathrm{mg})$ for luteal phase support starting from the day of oocytes retrieval. Serum B-hCG level was assessed 14 days after embryo transfer and the result was not regarded as being positive except for values exceeding $5 \mathrm{IU} /$ litre.

Clinical pregnancies confirmed by the presence of a gestational sac with fetal cardiac activity on vaginal ultrasound examination 6 weeks after embryo transfer.

Patients in the control group were subjected to :

\section{1) Verbal consent} study

Consent was obtained from the patients included in the 2) History taking as previously mentioned in unexplained infertility group.

3) Clinical examination as previously mentioned in unexplained infertility group

4) Imaging: ultrasound to assess pelvis. 
5) Ultrasound Technique: Color Doppler examination of the uterine, ovarian and subendometrial arteries and $\mathrm{PI} / \mathrm{RI}$ measurements were performed and average values were recorded.

This US examination was done in midluteal phase (peri-implantation) period from day 19 to day 21 .

\section{STATISTICAL ANALYSIS}

Data were collected, revised, coded and entered to the Statistical Package for Social Science (IBM SPSS) version 23. The quantitative data were presented as mean, standard deviations and ranges when parametric. Also qualitative variables were presented as number and percentages. The comparison between two independent groups regarding quantitative data with parametric distribution was done by using Independent t-test. Pearson correlation coefficients were used to assess the correlation between two quantitative parameters in the same group. Receiver operating characteristic curve (ROC) was used to assess the best cut off point for uterine, ovarian, subendometrial PI and RI as predictors for pregnancy outcome and to differentiate between unexplained infertility group and fertile group. The confidence interval was set to $95 \%$ and the margin of error accepted was set to $5 \%$.

\section{RESULTS}

This study included 102 patients who were divided into 2 groups and met the inclusion criteria, UI infertility group and fertile control group.

This study showed that there was no statistically significant difference found between the two studied groups regarding age and BMI with $p$-value $=0.125$ and 0.361 , respectively (Table 1 ). Also, table 1 showed that the age of the studied group ranged from 20 and 36 years with a mean of 28.02 for control group and 29.25 for UI. The body mass index (BMI) ranged from 22.3-29.8 with a mean of 25.83 for control and ranged from 20.9-29.5 with mean of 25.43 for UI.

The study revealed that the mean serum FSH is 6.69, mean serum LH is 5.60 , mean serum TSH is 2.38 , mean serum prolactin is 16.19 and mean serum E2 is 55.00 and mean endometrial thickness is 11.54 (Table 2).

The study showed that there was statistically significant increase in uterine and subendometrial PI in unexplained infertility group than control group and significant increase in uterine, ovarian and subendometrial RI in unexplained infertility group than control fertile group; while the ovarian artery PI did not statistically differ between unexplained infertility and control group (Table 3 ). This clinically means that there is impaired uterine, ovarian and subendometrial blood flow in unexplained infertility compared to fertile group.

The study showed that chemical and clinical pregnancy rates were the same $(23.5 \%)$ (Table 4$)$.

The study revealed that there was statistically significant decrease in uterine PI in positive chemical pregnancy cases than negative pregnancy cases with $p$-value $<0.001$. However, there was statistically significant decrease in ovarian and subendometrial PI and RI in positive chemical pregnancy cases than negative chemical pregnancy cases. But there was no statistically significant decrease in uterine RI in positive chemical pregnancy cases than negative pregnancy cases (Table 5).

The cut off values of PI and RI between unexplained infertility and fertile group are $\geq 1.88, \geq 0.8$ for uterine artery, and $\geq 0.87, \geq 0.53$ for subendometrial arteries and $\geq 0.59$ for ovarian artery RI (Fig. 1).

The cut off values of PI and RI between pregnant and non-pregnant outcome among unexplained infertility group are $\leq 1.13, \leq 0.59$ for ovarian artery and $\leq 0.89, \leq 0.56$ for subendometrial arteries and uterine artery PI $\leq 2.43$ (Fig. 2). 
Table 1: Demographic and characteristics of control and cases groups

\begin{tabular}{|c|c|c|c|c|c|c|}
\hline & & $\begin{array}{c}\text { Control group } \\
\text { No. }=51\end{array}$ & $\begin{array}{l}\text { Study group } \\
\text { No. }=51\end{array}$ & $\begin{array}{c}\text { Test } \\
\text { value• }\end{array}$ & P-value & Sig. \\
\hline \multirow{4}{*}{ Age } & Mean \pm SD & $28.02 \pm 4.56$ & $29.25 \pm 3.43$ & & & \\
\hline & & & & -1.546 & 0.125 & NS \\
\hline & Range & $20-36$ & $23-36$ & & & \\
\hline & Mean \pm SD & $25.83 \pm 2.16$ & $25.43 \pm 2.20$ & & & \\
\hline \multirow[t]{2}{*}{ BMI } & Range & $22.3-29.8$ & $20.9-29.5$ & 0.917 & 0.361 & NS \\
\hline & & $26-32$ & $26-32$ & & & \\
\hline
\end{tabular}

$P$-value $>0.05$ : Non significant (NS); P-value $<0.05$ : Significant (S); P-value $<0.01$ : highly significant (HS)

$\bullet$ Independent t-test

Table 2: Basal hormonal profile, endometrial thickness, number and quality of embryos transferred and day of ET among UI patients

\begin{tabular}{|c|c|c|}
\hline & & Cases group \\
\hline & & No. $=51$ \\
\hline \multirow[t]{2}{*}{ Day 3 FSH } & Mean \pm SD & $6.69 \pm 2.16$ \\
\hline & Range & $3.8-12.8$ \\
\hline \multirow[t]{2}{*}{ Day $3 \mathrm{LH}$} & Mean \pm SD & $5.60 \pm 2.40$ \\
\hline & Range & $2-14$ \\
\hline \multirow[t]{2}{*}{ Day 3 E2 } & $\operatorname{Mean} \pm \mathrm{SD}$ & $55.00 \pm 20.49$ \\
\hline & Range & $18-93$ \\
\hline \multirow[t]{2}{*}{$\mathrm{TSH}$} & Mean \pm SD & $2.38 \pm 1.08$ \\
\hline & Range & $0.76-5.36$ \\
\hline \multirow[t]{2}{*}{ Prolactin } & Mean \pm SD & $16.19 \pm 8.09$ \\
\hline & Range & $4-50$ \\
\hline \multirow{2}{*}{$\begin{array}{l}\text { Endometrial thickness }(\mathrm{mm}) \\
\text { on day of embryo transfer }\end{array}$} & Mean \pm SD & $11.54 \pm 2.33$ \\
\hline & Range & $8-19$ \\
\hline \multirow[t]{2}{*}{ N. of embryos transferred } & Mean \pm SD & $2.16 \pm 0.73$ \\
\hline & Range & $1-3$ \\
\hline Quality & Grade 1 & $51(100.0 \%)$ \\
\hline \multirow[t]{2}{*}{ Day of ET } & Mean \pm SD & $3.27 \pm 0.70$ \\
\hline & Range & $3-5$ \\
\hline
\end{tabular}


Table 3: Comparison between control group and cases group regarding uterine, ovarian and subendometrial PI and RI done at preimplantation period for control and day of embryo transfer for cases

\begin{tabular}{|c|c|c|c|c|c|c|}
\hline & & $\begin{array}{l}\text { Control } \\
\text { group }\end{array}$ & $\begin{array}{l}\text { Study } \\
\text { group }\end{array}$ & $\begin{array}{c}\text { Test } \\
\text { value }\end{array}$ & P-value & Sig. \\
\hline \multirow[t]{3}{*}{ Uterine PI } & Mean \pm SD & $1.71 \pm 0.22$ & $2.67 \pm 0.49$ & & & \\
\hline & & & & -12.910 & 0.000 & HS \\
\hline & Range & $1.52-2.72$ & $1.4-4.86$ & & & \\
\hline \multirow[t]{3}{*}{ Uterine RI } & Mean \pm SD & $0.77 \pm 0.04$ & $0.94 \pm 0.29$ & & & \\
\hline & & & & -3.920 & 0.000 & HS \\
\hline & Range & $0.65-0.89$ & $0.72-2.33$ & & & \\
\hline \multirow[t]{3}{*}{ Ovarian PI } & Mean \pm SD & $0.96 \pm 0.28$ & $1.00 \pm 0.14$ & & & \\
\hline & & & & -0.955 & 0.342 & NS \\
\hline & Range & $0.42-1.23$ & $0.47-1.3$ & & & \\
\hline \multirow[t]{3}{*}{ Ovarian RI } & Mean \pm SD & $0.58 \pm 0.06$ & $0.62 \pm 0.08$ & & & \\
\hline & & & & -2.642 & 0.010 & S \\
\hline & Range & $0.39-0.84$ & $0.34-0.94$ & & & \\
\hline \multirow[t]{3}{*}{ Subendometrial PI } & Mean \pm SD & $0.89 \pm 0.25$ & $0.99 \pm 0.25$ & & & \\
\hline & & & & -2.060 & 0.042 & S \\
\hline & Range & $0.77-2.55$ & $0.75-2.39$ & & & \\
\hline \multirow[t]{3}{*}{ Subendometrial RI } & Mean \pm SD & $0.54 \pm 0.07$ & $0.59 \pm 0.07$ & & & \\
\hline & & & & -3.322 & 0.001 & HS \\
\hline & Range & $0.43-1$ & $0.51-1$ & & & \\
\hline
\end{tabular}

$P$-value $>0.05$ : Non significant (NS); P-value $<0.05$ : Significant (S); P-value $<0.01$ : highly significant (HS)

$\bullet:$ Independent t-test

Table 4: Chemical and clinical pregnancy outcome among the UI patients undergoing ICSI

\begin{tabular}{lccc} 
& & No. $=51$ & $\%$ \\
\hline & Negative & 39 & $76.5 \%$ \\
Chemical pregnancy & Positive & 12 & $23.5 \%$ \\
& Single & 10 & $19.6 \%$ \\
& Twin & 2 & $3.9 \%$ \\
\hline
\end{tabular}


Table 5: Comparison between pregnant and non-pregnant as regard uterine, ovarian and subendometrial PI and RI in patients who have undergone ICSI (No. $=51)$

\begin{tabular}{|c|c|c|c|c|c|c|}
\hline \multicolumn{7}{|c|}{ Chemical pregnancy } \\
\hline & & Negative & Positive & value & P-value & Sig. \\
\hline & & No. $=39$ & No. $=12$ & & & \\
\hline \multirow{3}{*}{ Uterine PI } & Mean \pm SD & $2.82 \pm 0.42$ & $2.17 \pm 0.33$ & \multirow{3}{*}{4.854} & \multirow{3}{*}{0.000} & \multirow{3}{*}{ HS } \\
\hline & & & & & & \\
\hline & Range & $2.41-4.86$ & $1.4-2.53$ & & & \\
\hline \multirow{3}{*}{ Uterine RI } & Mean \pm SD & $0.97 \pm 0.32$ & $0.81 \pm 0.04$ & \multirow{3}{*}{1.720} & \multirow{3}{*}{0.092} & \multirow{3}{*}{ NS } \\
\hline & & & & & & \\
\hline & Range & $0.72-2.33$ & $0.73-0.87$ & & & \\
\hline \multirow{3}{*}{ Ovarian PI } & Mean \pm SD & $1.12 \pm 0.11$ & $1.02 \pm 0.19$ & \multirow{3}{*}{2.377} & \multirow{3}{*}{0.021} & \multirow{3}{*}{ S } \\
\hline & & & & & & \\
\hline & Range & $0.82-1.3$ & $0.47-1.17$ & & & \\
\hline \multirow{3}{*}{ Ovarian RI } & Mean \pm SD & $0.63 \pm 0.08$ & $0.57 \pm 0.08$ & \multirow{3}{*}{2.456} & \multirow{3}{*}{0.018} & \multirow{3}{*}{ S } \\
\hline & & & & & & \\
\hline & Range & $0.48-0.94$ & $0.34-0.64$ & & & \\
\hline \multirow{3}{*}{ Subendometrial PI } & Mean \pm SD & $1.03 \pm 0.27$ & $0.85 \pm 0.05$ & \multirow{3}{*}{2.326} & \multirow{3}{*}{0.024} & \multirow{3}{*}{ S } \\
\hline & & & & & & \\
\hline & Range & $0.84-2.39$ & $0.75-1.05$ & & & \\
\hline \multirow{3}{*}{ Subendometrial RI } & Mean \pm SD & $0.61 \pm 0.07$ & $0.54 \pm 0.02$ & \multirow{3}{*}{3.201} & \multirow{3}{*}{0.002} & \multirow{3}{*}{ HS } \\
\hline & & & & & & \\
\hline & Range & $0.52-1$ & $0.51-0.58$ & & & \\
\hline
\end{tabular}

$P$-value $>0.05$ : Non significant (NS); P-value $<0.05$ : Significant (S); $P$-value $<0.01$ : highly significant (HS)

$\bullet:$ Independent t-test 


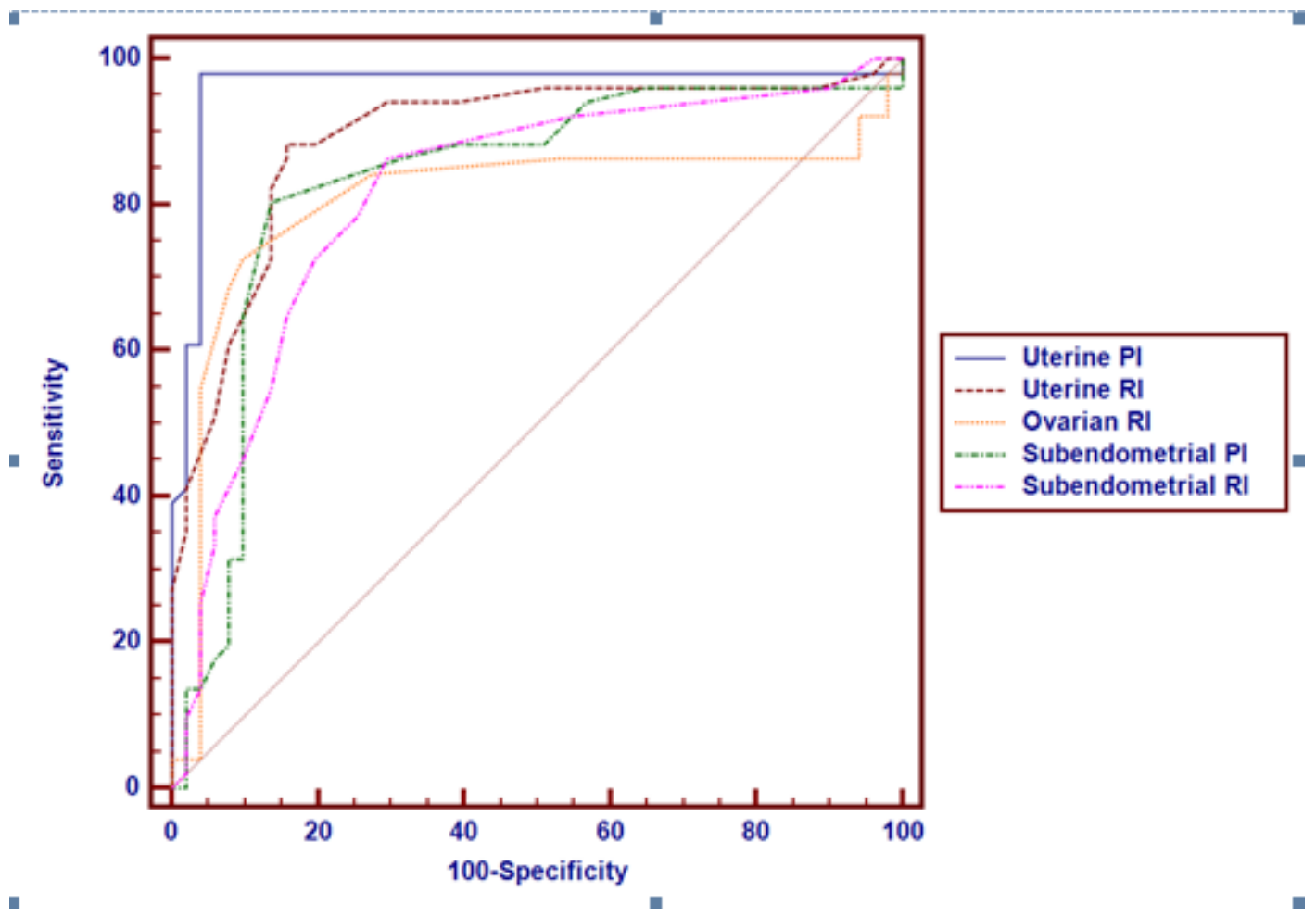

Fig. 1: Receiver operating characteristic (ROC) curve of uterine, ovarian and subendometrial PI and RI in differentiation between control group and unexplained infertility group

\begin{tabular}{lccccccc}
\hline & Parameter & AUC & Cut of Point & Sensitivity & Specificity & PPV & NPV \\
\hline \multirow{2}{*}{$\begin{array}{l}\text { Uterine } \\
\text { PI }\end{array}$} & 0.962 & $>1.88$ & 98.04 & 96.08 & 96.2 & 98.0 \\
Ovarian & RI & 0.892 & $>0.8$ & 88.24 & 84.31 & 84.9 & 87.8 \\
\multirow{2}{*}{ Subendometrial } & RI & 0.806 & $>0.59$ & 72.55 & 90.20 & 88.1 & 76.7 \\
& PI & 0.831 & $>0.87$ & 80.39 & 86.27 & 85.4 & 81.5 \\
\hline
\end{tabular}




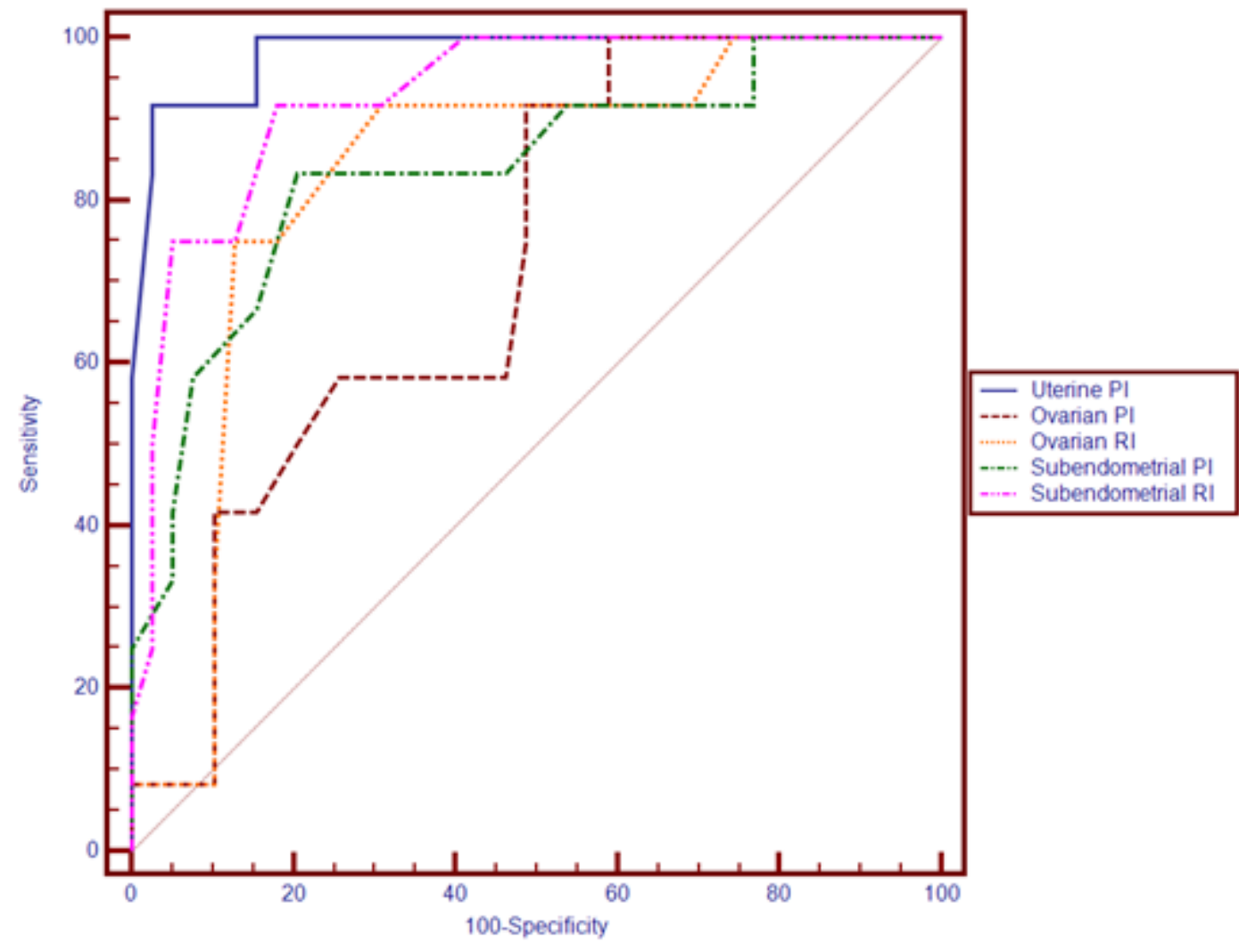

Fig. 2: ROC curve of uterine, ovarian and subendometrial PI and RI in differentiation between negative and positive pregnancy

\begin{tabular}{|c|c|c|c|c|c|c|c|}
\hline & Parameter & AUC & Cut of Point & Sensitivity & Specificity & PPV & NPV \\
\hline \multirow{2}{*}{ Uterine } & PI & 0.982 & $\leq 2.43$ & 91.67 & 97.44 & 91.7 & 97.4 \\
\hline & PI & 0.722 & $\leq 1.13$ & 91.67 & 51.28 & 36.7 & 95.2 \\
\hline Ovarian & RI & 0.826 & $\leq 0.59$ & 75.00 & 87.18 & 64.3 & 91.9 \\
\hline \multirow[b]{2}{*}{ Sub endometrial } & PI & 0.838 & $\leq 0.89$ & 83.33 & 79.49 & 55.6 & 93.9 \\
\hline & RI & 0.927 & $\leq 0.56$ & 91.67 & 82.05 & 61.1 & 97.0 \\
\hline
\end{tabular}




\section{DISCUSSION}

This study was a prospective case control study conducted in the Department of Obstetrics and Gynecology Fertility and IVF unit in Ain-Shams University Hospital, Egypt during the period from September 2017 to July 2019. It included two groups of patients; one group with unexplained infertility attending the Assisted Reproduction Unit and the other is control fertile group, each group consists of 51 women. The aim of the study was to compare ovarian, uterine artery and subendometrial vessels resistance of patients diagnosed as UI undergoing ICSI in the periimplantation period and control group diagnosed as fertile by having at least one livebirth.

Color Doppler examination of the subendometrial arteries was performed within 1-mm area parallel to the myometrium endometrium border Descending branches of uterine artery was determined at the level of the internal os of cervix uteri. Right and left uterine artery pulsatility index (PI) and resistance index (RI) was calculated and average values were recorded.

Both ovarian arteries were evaluated at ovarian hilus for ovarian arterial study. PI/RI measurements were performed for ovarian artery at ovarian hilus distant from ovarian surface and follicular wall surface. Average values will be recorded.

This ultrasound examination was done at day of embryo transfer of ICSI technique for UI group and peri-implantation period for control group. The age of the studied groups ranged from 20 to 36 years with a mean $28.02 \pm 4.56$ for control group and mean of $29.25 \pm 3.43$ for UI group. The body mass index (BMI) ranged from 22.3 to 29.8 with a mean of $25.83 \pm 2.16$ for control group and ranged from 20.9 to 29.5 with a mean of $25.43 \pm 2.20$.

All cycles used long GnRH agonist protocol embryo transfer carried out according to number of embryos and embryo quality on day 3 or day 5 post ovum pick up according to local protocol of IVF unit all with fresh embryos. For UI group Doppler parameters were evaluated on the day of ET. On evaluating the subendometrial blood flow, the mean PI was $0.99 \pm 0.25$ and the mean RI was $0.59 \pm 0.7$.

An average of both right and left uterine arteries' blood flows was taken so that it can be clinically significant. The mean uterine PI was $2.67 \pm 0.49$ and the mean RI was $0.94 \pm 0.29$. The mean ovarian PI was $1.00 \pm 0.14$ and the mean RI was $0.62 \pm 0.08$.
In control fertile group, Doppler parameters were evaluated in peri-implantation period from day 19 to day 21 , on evaluating the sub-endometrial blood flow, the mean PI was $0.89 \pm 0.25$ and the mean RI was $0.54 \pm 0.07$. The mean uterine PI was $1.71 \pm 0.22$ and the mean RI was $0.77 \pm 0.04$. The mean ovarian PI was $0.96 \pm 0.28$ and the mean RI was $0.58 \pm 0.06$.

The above mentioned parameters were compared as regard the two groups and the pregnancy outcome. There was no statistically significant difference between the two groups (unexplained infertility group and control fertile group) as regard the age $(p$-value $=0.125)$ and BMI $(p$-value $=0.361)$.

There was statistically significant difference between the unexplained infertility group and control fertile group as regard uterine and subendometrial artery PI, RI and ovarian RI in which resistance indexes were significantly higher in the UI group than control group.

The cut off values of PI, RI between unexplained infertility and fertile group are $\geq 1.88, \geq 0.8$ for uterine artery and $\geq 0.87, \geq 0.53$ for subendometrial arteries and $\geq 0.59$ for ovarian artery RI.

Among the unexplained infertility undergoing ICSI patients the clinical pregnancy rate is $23.5 \%$. There was no statistically significant difference between the two groups (pregnant group and non-pregnant group) as regard the age, BMI, duration of infertility or the basal hormonal profile and endometrial thickness.

There was statistically significant difference between the two groups (pregnant group and non-pregnant group) as regard uterine artery $\mathrm{PI}(p$-value $=0.000)$, ovarian artery PI $(\mathrm{p}$-value $=0.021)$, ovarian artery RI ( $p$ value $=0.018)$, subendometrial arteries PI $(p$-value $=0.024)$ and subendometrial arteries RI ( $p$-value $=0.002)$. However, there was no statistically significant difference between both groups as regard uterine artery RI with $p$ value $=0.092$.

These results agree with Zebitay et al. (2015) ${ }^{[14]}$ who compared ovarian, uterine and spiral artery resistance in the three studied groups of patients unexplained infertility $n=70$, tubal factor infertility $n=75$ and fertile $n=72$. He found that both TFI and control groups UA PI values were significantly lower than the UI group's PI values and the highest UA RI values were found in UI group and the lowest values were in the control group. UI and TFI groups' OA PI/RI values were significantly 
higher than the control group, both the control and TFI groups' SA PI/RI values were significantly lower than UI group's PI/RI values.

UI patients' uterine and spiral arteries PI values $\geq 1.86$ and $\geq 0.85$, RI values $\geq 0.80$ and $\geq 0.53$ can be used as a valuable test showing reduced uterine perfusion. Ovarian artery PI values $\geq 0.96$ and RI values $\geq 0.58$ can be used as tests showing decreased ovarian perfusion in patients with TFI. In these patients, embryo cryopreservation can be considered.

Also, this study results agree with El-Mazny et al. (2013) ${ }^{[5]}$ who compared 40 women with unexplained infertility with 40 fertile parous controls by measuring the endometrial thickness and volume, uterine artery pulsatility index (PI) and resistance index (RI), endometrial and subendometrial 3D power Doppler vascularization index (VI), flow index (FI) and vascularization flow index (VFI) and serum estradiol and progesterone levels in peri-implantation and found that the uterine artery PI and RI were significantly increased and the endometrial VI, FI and VFI and subendometrial VI, FI and VFI were significantly decreased in the unexplained infertility group. The endometrial thickness and volume and serum estradiol and progesterone levels, however, were not significantly different between the two groups.

Also, these study results are in consistent with Wang et al. (2010) ${ }^{[13]}$ who recruited 182 women (38 year or younger) undergoing IVF-ET endometrial thickness, echo pattern and blood flow on transvaginal ultrasonography were recorded eight hours prior to hCG administration. The patients were divided into three groups; A $(n=10)$ with undetectable endometrial blood flow, $\mathrm{B}(\mathrm{n}=82)$ with sub-endometrial blood flow and $C(n=90)$ with both endometrial and subendometrial blood flow. According to IVF ET outcomes, all patients were re-divided into three groups; 1 - nonpregnancy $(\mathrm{n}=92), 2$ - intrauterine pregnancy with live fetus $(n=70)$ and 3 others $(n=20)$ including biochemical pregnancy, embryonic diapause, ectopic pregnancy and miscarriage. Intrauterine pregnancy with live fetus in group $\mathrm{C}(62.2 \%)$ was much higher than that in group $\mathrm{A}$ and $\mathrm{B}(0 \%$ and $17.1 \%$, p less than or equal to 0.001$)$. The implantation rate $(33.2 \%)$ was much higher than that in groups $\mathrm{A}$ and $\mathrm{B}(0 \%$ and $19.90 \%$, less than or equal to 0.001 ). The pulsatility index, resistance index and S/D of endometrial spiral arteries were $0.1+/-0.2,0.6+/-0.1$ and $2.5+/-0.4$ in group 2, which were much lower than those in groups 1 and 3. It was concluded that the patients with detectable endometrial blood flow had higher clinical pregnancy rates and implantation rates.
However, this study results disagree with Schild et al. (2001) ${ }^{[11]}$ who recruited 135 patients in IVF program measured uterine artery blood flow on the day of oocyte retrieval resulted in overall implantation rate was $23.7 \%$ per cycle, subendometrial blood flow was detected in $113(83.7 \%)$ cases with pregnancy occurring in $21.2 \%$. Mean spiral artery pulsatility index values were $1.12 \pm 0.28$ and $1.21 \pm 0.27$ for nonconception and conception cycles, respectively.

Thus, concluded that neither Doppler sonography of the spiral or uterine arteries nor measurement of the endometrial thickness or volume allowed a reliable prediction of subsequent IVF outcome.

Also, these results disagree with Isaksoon et al. (2003) who compared uterine and spiral artery pulsatility index and peak systolic velocity in women with unexplained infertility $(\mathrm{n}=20)$ or tubal infertility $(\mathrm{n}=18)$, his study resulted in clinical pregnancy was achieved in $8 / 20(40 \%)$ women with unexplained and $6 / 18(33.3 \%)$ women with tubal infertility with in-vitro fertilization treatment. There were no differences in the uterine artery pulsatility index or peak systolic velocity during the spontaneous or the in-vitro fertilization cycle between the two groups. The impedance to blood flow in the uterine or spiral artery did not differ between women conceiving with in-vitro fertilization-embryo transfer and those who did not.

Abdel Kader et al. (2016) ${ }^{[1]}$ conducted a prospective observational study including 100 women undergoing IVF/ICSI treatment at a University based IVF center. Endometrial thickness, morphology and subendometrial blood flow were assessed using transvaginal ultrasound on the day of hCG. RI, PI and $\mathrm{S} / \mathrm{D}$ ratio of sub-endometrial blood flow were measured. There was no statistically significant difference in the Doppler indices of the sub-endometrial blood vessels between pregnant and non-pregnant women.

Kim et al. (2014) ${ }^{[7]}$ who conducted a prospective observational study that enrolled 236 nulliparous women who underwent a first IVF-ET using a GnRH long protocol with stimulation by recombinant FSH (rFSH). Color Doppler ultrasound was performed on the day of embryo transfer. Main outcomes were pulsatility index (PI), resistance index (RI), systolic/ diastolic ratio (S/D) of the uterine artery. Measurements were analysed relative to IVF-ET outcome (pregnant vs. non-pregnant). Uterine artery PI, RI, or S/D scores $(\mathrm{p}=0.256, \mathrm{p}=0.527, \mathrm{p}=0.365)$ didn't have statistically significant difference between pregnant and non- pregnant groups. 
Arterial resistance can be expected to rise further in infertility treatments. Embryo cryopreservation can be considered as a routine practice to increase the success of the implantation of embryos and to be protected from possible adverse effects of superovulation treatment. In the following cycles, embryo transfer with endometrial preparation can protect the adverse effects of the superovulation treatment on vascular resistance. It can increase the pregnancy rates.

UI patient's uterine and subendometrial arteries PI values $\geq 2.43$ and $\geq 0.89$, subendometrial arteries RI values $\geq 0.56$ and ovarian artery $\mathrm{PI} \geq 1.13, \mathrm{RI} \geq 0.59$ can be used as a valuable test showing reduced uterine perfusion. UI patients who will undergo IVF treatment having PI and RI values higher than these values, may have the embryo transfer in the cycles following the ovulation stimulation. In these patients, treatment can be planned to reduce the vascular resistance.

It seems that the controversial results which have reported in different studies are due to the differences in patients' characteristics, study designs, population sizes. The protocol of ovarian stimulation, the embryo transfer protocols, the day of ultrasound evaluation and the use of different Doppler parameters.

\section{CONCLUSION}

Doppler parameters are useful tools to assess endometrial receptivity in unexplained infertility patients undergoing ICSI. This study found that uterine, ovarian, subendometrial arteries pulsatility index (PI) and resistance index RI measured on the day of embryo transfer higher than fertile women and have value in judging endometrial receptivity and predict the final outcome of IVF/ ICSI- ET. Uterine, ovarian and subendometrial blood flow assessed by measuring PI, RI of these arteries was impaired in unexplained infertility patients compared to fertile women. Also, uterine arteries PI, ovarian and subendometrial arteries PI, RI differ in pregnancy outcome in unexplained infertility patients undergoing ICSI.

\section{CONFLICT OF INTEREST}

There are no conflicts of interests.

\section{REFERENCES}

1. Abdel Kader M, Abdelmeged A, Mahran A, et al. The usefulness of endometrial thickness, morphology and vasculature by $2 \mathrm{D}$ Doppler ultrasound in prediction of pregnancy in IVF/ICSI cycles. The Egyptian Journal of Radiology and Nuclear Medicine. 2016; 47(1): 3416-.

2. Abma J, Chandra A, Mosher W, et al. Fertility, family planning, and women's health: new data from the 1995 National Survey o Family Growth. Vital Health Stat, 1997; 23(1).

3. Assisted Reproductive Technologies Review Committee. Report of the independent review of assisted reproductive technologies. 2006. http: // www. Health. Gov. au/ internet/ main/ publishing. nsf/ Content/ 79D96DD80F01073ECA257. BF0001 C1ABB/ \$File/ artrc report.pdf. Accessed 29 Apr 2016.

4. Gardner AW. Textbook of Assisted Reproductive Technologies: Laboratory and Clinical Perspectives. 2008.

5. El-Mazny A, Abou-Salem $\mathrm{N}$ and Elshenoufy $\mathrm{H}$. Doppler study of uterine hemodynamics in women with unexplained infertility. Eur J Obstet Gynecol Reprod Biol 2013; 171: 84-7.

6. Fanchin R, Ayoubi JM, Righini C, et al. Uterine contractility decreases at the time of blastocyst transfers. Human Reproduction. 2001; 16(6): 1115-9.

7. Kim A, Jung H, Choi WJ, et al. Detection of endometrial and subendometrial vasculature on the day of embryo transfer and prediction of pregnancy during fresh in vitro fertilization cycles. Taiwanese journal of obstetrics \& gynecology. 2014; 53(3): 360-5.

8. Mascarenhas MN, Cheung H, Mathers CD, et al. Measuring infertility in populations: constructing a standard definition for use with demographic and reproductive health surveys. Popul Health Metr. 2012; 10(1): 1-11.

9. Ng EH, Chan CC, Tang OS, et al. Factors affecting endometrial and subendometrial blood flow measured by three-dimensional power Doppler ultrasound during IVF treatment. Hum Reprod 2006; 21: 1062-9. 
10. Practice Committee of the American Society for Reproductive M, Practice Committee of the Society for Assisted Reproductive T. Guidelines on number of embryos transferred. Fertility and sterility. 2009; 92(5): 1518-9.

11. Schild RL, Knobloch C, Dorn C, et al. Endometrial receptivity in an in vitro fertilization program as assessed by spiral artery blood flow, endometrial thickness, endometrial volume, and uterine artery blood flow. Fertil Steril 2001; 75: 361-6.

12. Steer CV, Campbell S, Pampiglione JS, et al. Transvaginal color flow imaging of the uterine arteries during the ovarian and menstrual cycles. Hum Reprod 1990; 5: 391-395.
13. Wang L, Qiao J, Li R, et al. Role of endometrial blood flow assessment with color Doppler energy in predicting pregnancy outcome of IVF-ET cycles. Reprod. Biol. Endocrinol, 2010; 8: 122.

14. Zebitay AG, Tutumlu M, Verit FF, et al. A comparative analysis of arterial blood flow in unexplained infertility, tubal infertility and fertile groups. Gynecol Endocrinol 2015; 4(5): 61-74.

15. Zegers-Hochschild F, Adamson GD, de Mouzon $\mathrm{J}$, et al. International committee for monitoring assisted reproductive technology (ICMART) and the World Health Organization (WHO) revised glossary of ART terminology, 2009. Fertil Steril. 2009; 92: 1520-4. 